\title{
Subpectoral pacemaker implant after repeated pocket complications due to "senile pruritus"
}

\author{
Andreea-Maria Ursaru ${ }^{1}$, Andreea-Mihaela Ignat*,1, Dana Corduneanu², Gabriel \\ Mazilu $^{3}$, Antoniu Octavian Petriș ${ }^{1,4}$, Dan-Nicolae Tesloianu ${ }^{1}$ \\ ${ }^{1}$ Cardiology Department, "Sf. Spiridon" University Emergency Hospital, lasi, Romania, ${ }^{2}$ Internal \\ Medicine Department, "Sf. Spiridon" University Emergency Hospital, lasi, Romania, ${ }^{3}$ Plastic and \\ Reconstructive Microsurgery Department, "Sf. Spiridon" University Emergency Hospital, lasi, \\ Romania, "“'Grigore T. Popa" University of Medicine and Pharmacy, lasi, Romania
}

\begin{abstract}
Complications that can occur after pacemaker implant may be surgical or programming function related. We report a case of an 89-years old patient with slow atrial fibrillation, initially treated by external temporary pacing, and then by permanent pacemaker implant. The clinical course in the first months after procedure was uneventful, but after a half-year from the first admission, the patient addressed for pacemaker pocket infection, complaining of intense pruritus. After the drainage of the purulent secretions, the patient developed pocket hematoma, despite of Velpeau bandages and antipruritic drugs. Being a non-compliant patient with high risk of reinfection, it was decided, four days later after hematoma evacuation, to relocate the device on the same side, beneath pectoralis major muscle. This case presents two of the device pocket surgical complications (infection and hematoma) that occurred long after the implantation procedure due to senile pruritus.
\end{abstract}

Keywords: pacemaker pocket infection, permanent pacemaker, senile pruritus, pocket hematoma, subpectoral pacemaker implant

\section{Introduction}

Originally implanted in the anterior abdominal wall due to their size, as pacemaker dimensions decreased, the preferred implantation site became the anterior chest wall, on the pectoral fascia [1]. Sometimes subclavicular pocket is not suitable for all patients, such as pediatric population or cachectic patients, with very thin subcutaneous tissue, where device implant may become an unpleasant visible deformity $[2,3]$. In these cases, or in the presence of pocket complications such as infection, pain,

Received: February 2017; Accepted after review: June 2017; Published: June 2017.

${ }^{*}$ Corresponding author: Ignat Andreea-Mihaela, MD, Cardiology Department, "Sf. Spiridon" University Emergency Hospital, No. 1, Independentei Blv, lasi, Romania.

E-mail: andreea mihaela ignat@gmail.com palpability, or exposure, plastic surgeons may be consulted for reimplanting the device using a subpectoral approach [4].

\section{Case report}

An 89-years old patient, known with atrial fibrillation, was transferred in June 2016 to the Cardiology Department, after detecting on the electrocardiogram recording very slow heart rate (30 beats per min) (Figure 1). The patient was receiving chronic digitalis therapy, but the digoxin had been interrupted eight days before the moment of admission to our hospital. $\mathrm{He}$ was confused and temporo-spatial disoriented. Biochemical parameters revealed severe azotate retention, with a creatinine clearance of $20 \mathrm{ml}$ per minute.

Dilated cardiomyopathy, with moderate mitral and aortic regurgitations, depressed 
systolic function (an ejection fraction of $25 \%$ ), and medium secondary pulmonary hypertension (PAPS $55-60 \mathrm{mmHg}$ ), were the pathological findings at transthoracic echocardiography examination. His altered mental status and the slow heart rate entailed emergent temporary transvenous pacing (Figure 2A). After five days, at the attempt to stop temporary pacing, the patient maintained very slow heart rate - 35 beats per minute. Even though, it wasn't measured the digoxin serum level, after thirteen days (eight days before admission and five days during hospitalization) of digitalis interruption, it was concluded that the patient had enough time to eliminate the drug. Therefore, because the patient had a mean heart rate of 35 beats per minute, it was decided to perform a permanent unicameral VVIR pacemaker implant (Endurity Core SSI, St. Jude Medical), using right subclavian vein approach. Before procedure, written consent was obtained from his family. An active fixation lead-Trendil STS 2088TC/58, St. Jude Medical-was placed in the right ventricle (Fgure 2B - arrow), with acute threshold of $0.25 \mathrm{~V}$ at $0.4 \mathrm{~ms}$.

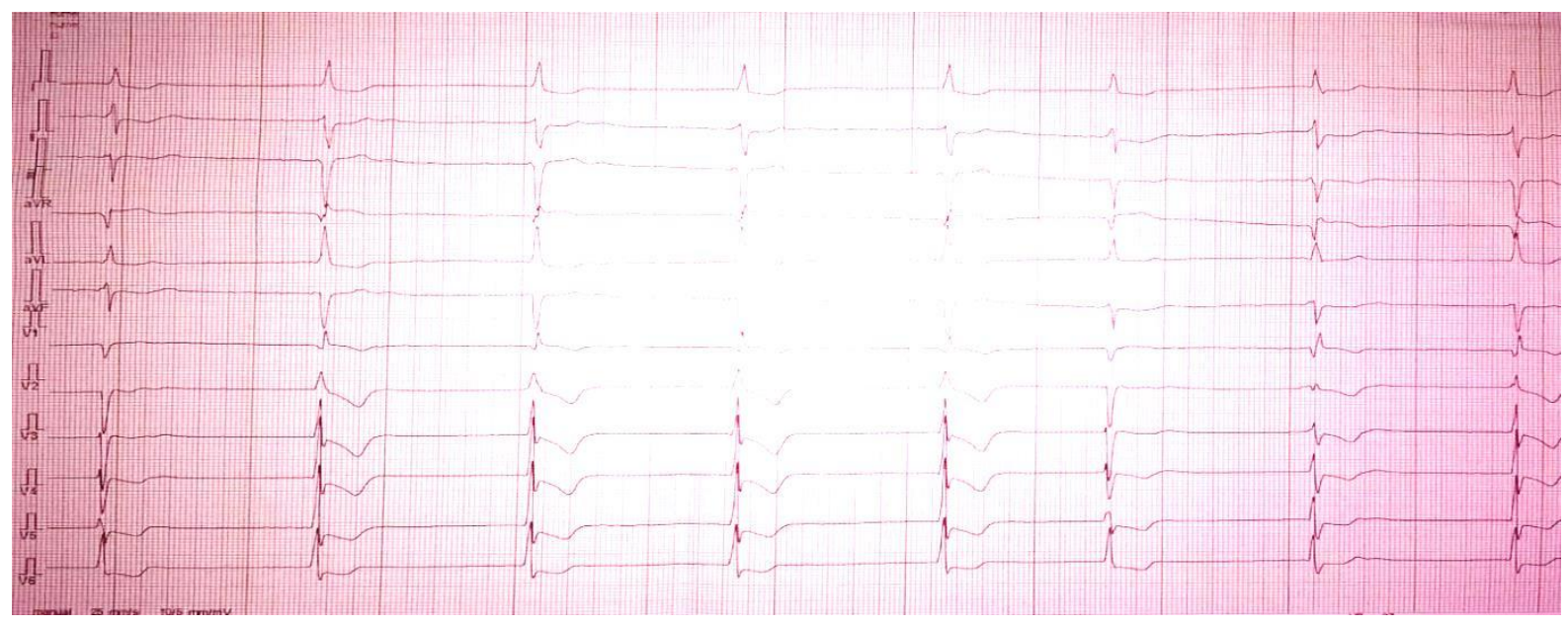

Fig. 1. Electrocardiogram recording showing slow atrial fibrillation with intermittent AV block with idioventricular escape rhythm with right bundle branch block pattern

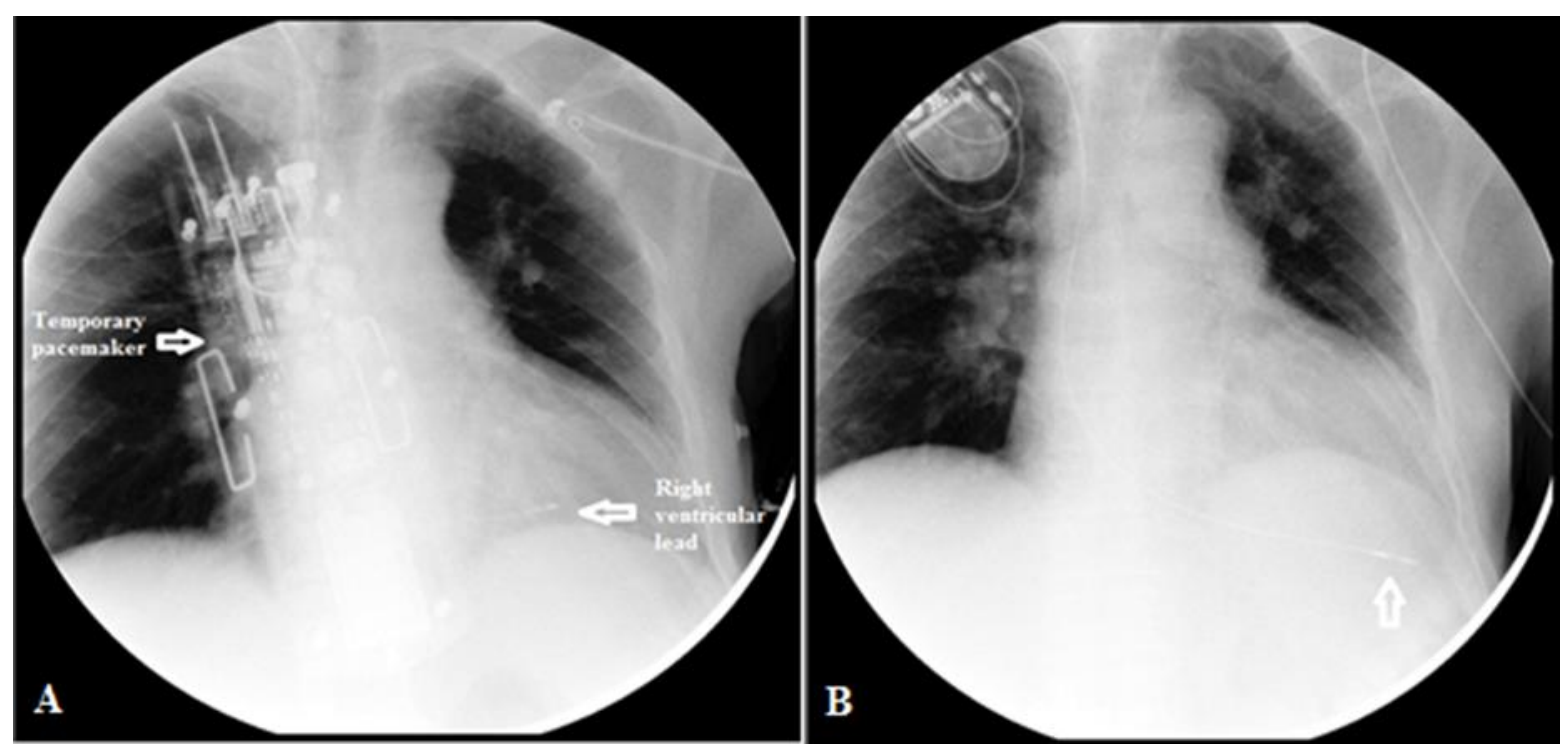

Fig. 2. Posteroanterior chest $X$-ray film: external temporary pacing $(A)$, followed by a permanent pacemaker implant (B) 
At discharge, the treatment plan included Dabigatran $75 \mathrm{mg}$ twice a day (his creatinine clearance being of $40 \mathrm{ml}$ per $\mathrm{min}$ ), Spironolactone $50 \mathrm{mg}$ daily, Furosemide 20 $\mathrm{mg}$ daily, Rilmenidine $1 \mathrm{mg}$ daily, Pantoprazole $40 \mathrm{mg}$ daily, and antibiotherapy for 10 days with Amoxicillin /Clavulanic Acid $875 \mathrm{mg} / 125 \mathrm{mg} \mathrm{q} 12 \mathrm{~h}$.

The patient returned for the two weeks and three months post-implant visits, having optimal electric parameters and a healed wound. Six months after the implant, the patient addressed again to our Department, for signs of inflammation at the pacemaker pocket (local tumefaction and hyperemia), with purulent secretions (Figure 3). At physical examination we also discovered multiple excoriations extended on his entire body, with areas of lichenification. The patient was apyretic and he was complaining of intense pruritus. His ECG revealed atrial fibrillation with a ventricular rate of 80 beats per minute, with intermittent periods of pacemaker dependency (Figure 4). The pacemaker interrogation showed $54 \%$ ventricular paced rhythm and $46 \%$ ventricular sensed rhythm. Biochemical laboratory investigations demonstrated a stable chronic kidney disease (creatinine clearance of $38 \mathrm{ml} / \mathrm{min}$ ). A nonspecific inflammatory syndrome (Creactive protein $2.86 \mathrm{mg} / \mathrm{dl}$, fibrinogen 423 $\mathrm{mg} / \mathrm{dl}$ ) and a moderate normocytic normochromic anemia (hemoglobin level of $9.7 \mathrm{~g} / \mathrm{dl}$ ), with normal iron deposits were found. The anemic syndrome was interpreted as a consequence of the chronic kidney disease.

Three sets of blood cultures were sampled, but the test results were negative. His echocardiographic measurements showed an improved ejection fraction (30\%), without any signs of endocarditis or ventricular lead infection.

Because of his advanced age and his noncompliance to treatment measures, it was decided to keep the same generator pocket, although the patient had indication to remove the whole pacemaker system, and to place a new one on the other side. After collecting secretion samples, a broad-spectrum antibiotherapy (Vancomycin $500 \mathrm{mg} \mathrm{q} 24 \mathrm{~h}$ and Ceftriaxone $1000 \mathrm{mg} \mathrm{q} 8 \mathrm{~h}$ ) was initiated, drug dosage being adapted to creatinine clearance. Next day, in the electrophysiology cardiology laboratory, under local anesthesia, two small incisions were performed at the pacemaker pocket. The intervention continued with the drainage of the purulent secretions and antiseptic lavage, followed by large sutures that closed the wound. The secretion test results indicated Staphylococcus aureus with high sensitivity to antibiotics. Therefore, prior antibiotherapy was replaced with Oxacilin $1000 \mathrm{mg} \mathrm{q} 6 \mathrm{~h}$, in concordance with antimicrobial susceptibility.

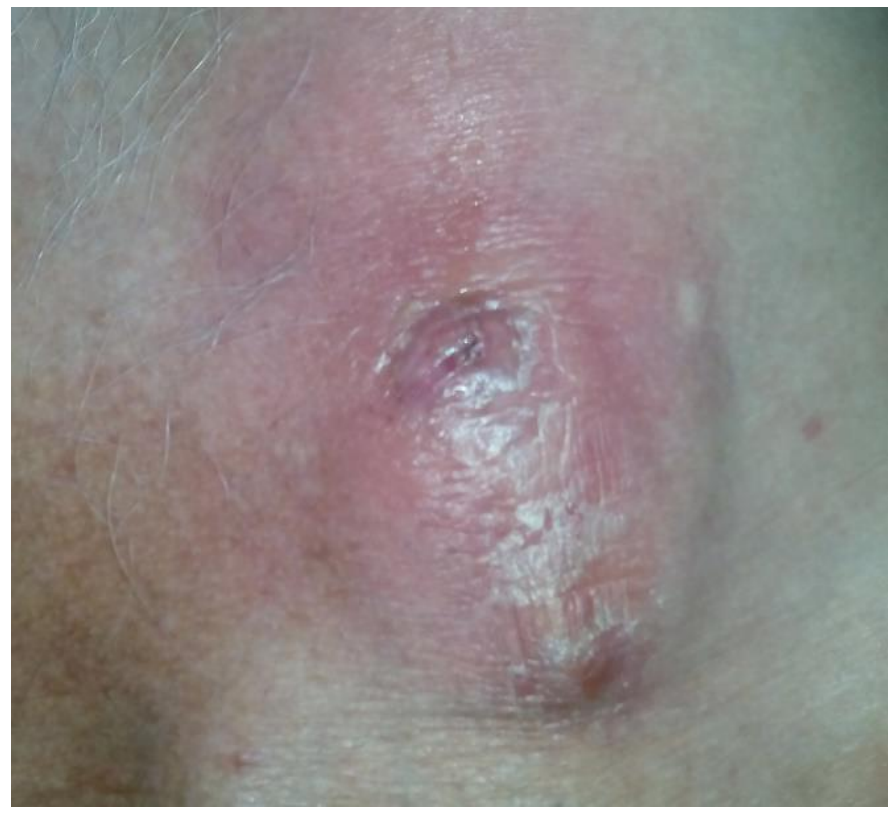

Fig. 3. Local infection at the pacemaker pocket 


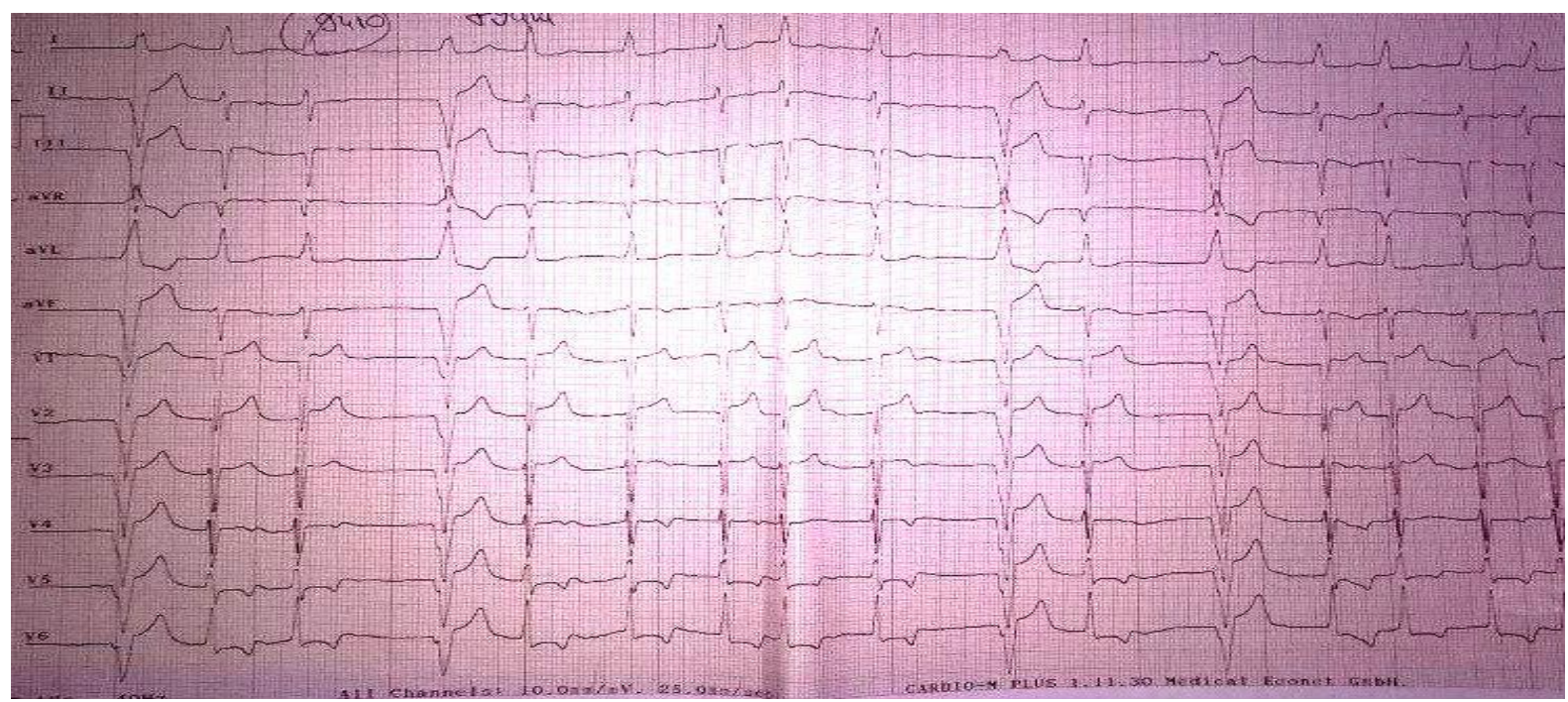

Fig. 4. Electrocardiogram recording: atrial fibrillation alternating with ventricular paced rhythm

After a dermatology evaluation, it was concluded that the patient suffered of senile pruritus. The recommended treatment included topic corticosteroids and emollients, together with systemic antihistamines. In the next ten days, the patient kept scratching the pocket area using his cane, despite different methods of bandage and correct dermatological treatment, even under sedation with Alprazolam. Repeated Velpeau bandages were performed, but each time he succeeded to untie it with his cane. Moreover, due to chronic pruritus, he developed pocket hematoma (Figure 5).

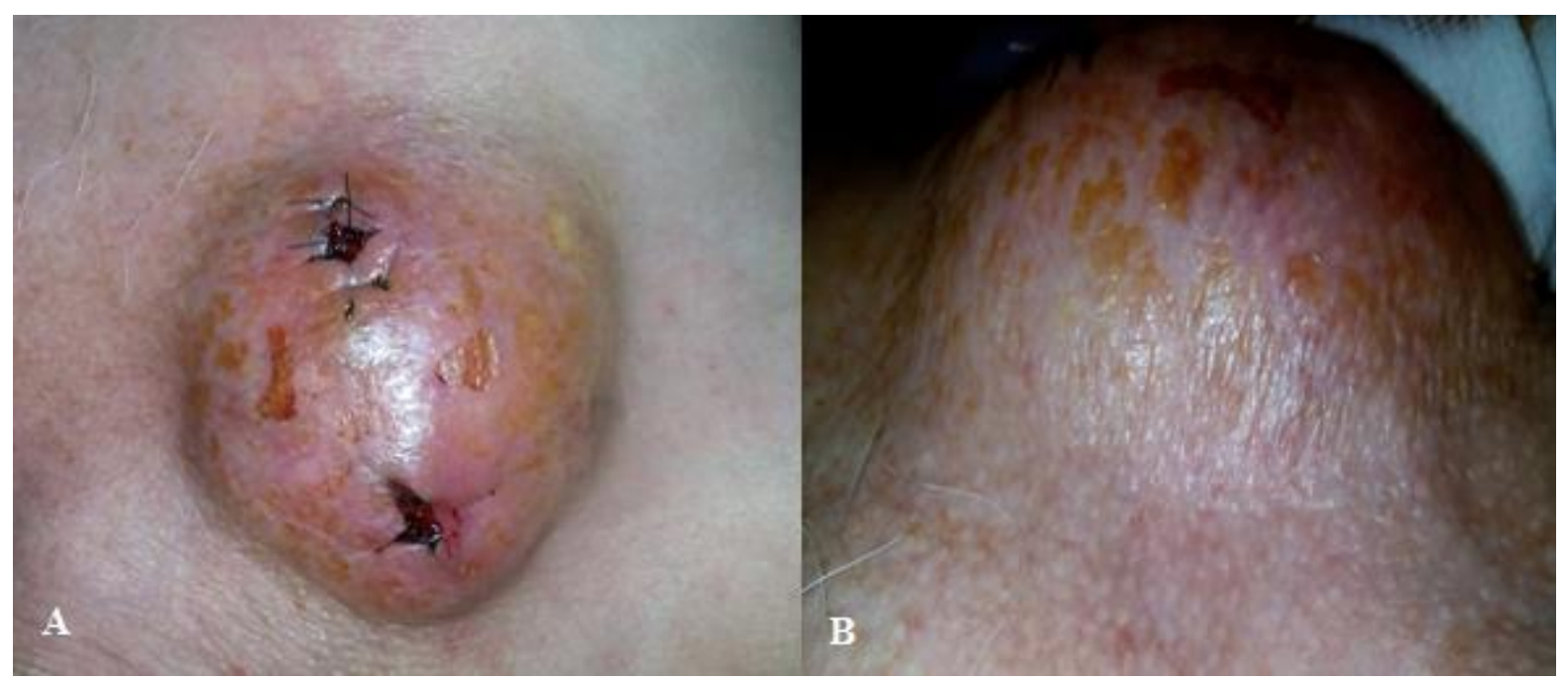

Fig. 5. Pacemaker pocket hematoma

Anticoagulant therapy with Enoxaparine was interrupted in the same day after the complication occurred. Because hematoma was expanding, being tense and painful, it demanded surgical revision to identify and arrest the site of bleeding. Assisted by a plastic surgeon, the sutures were removed for clots drainage. First, the pacemaker was extracted from its pocket, and then the blood clots were gentle evacuated. After lavage with antiseptic solutions, the generator was reintroduced in its prior location (Figure 6). 
Because there was a high risk of reinfection (persisting pruritus to a noncompliant patient), after another four days, we decided to relocate the device on the same side, beneath the pectoralis major muscle. Being no signs of deep local infection it was decided to use the same pacemaker pulse generator. The procedure demanded a pectoralis major muscle spreading incision. Using a blunt dissection, a subpectoral pocket was developed, without affecting pectoralis minor muscle. Once a satisfactory subpectoral pocket was created, the generator unit was placed in it (Figure 7A), and then the muscle was primarily closed over the device. Meticulous layered closure of the subcutaneous tissue was performed to obliterate dead space in the original superficial pocket (Figure 7B). Bacteriology report of the wound secretions revealed no growth.

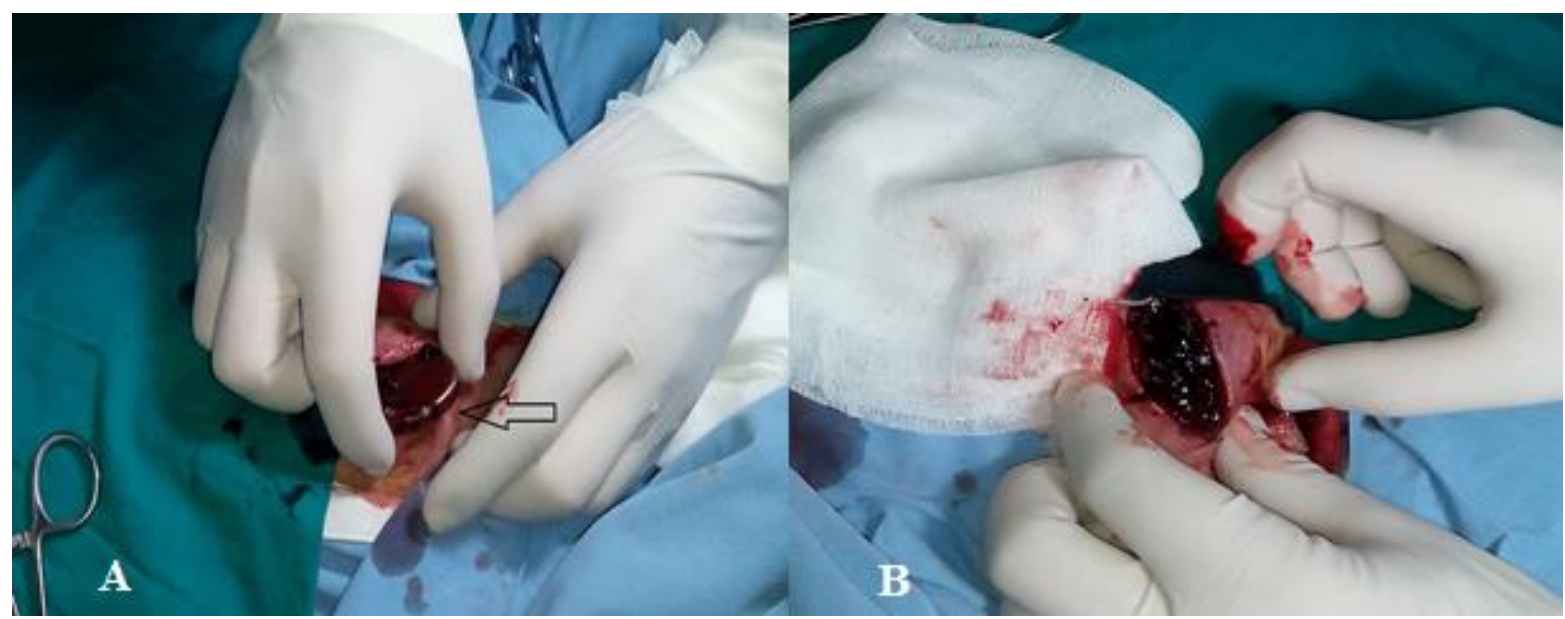

Fig. 6. Generator removal (A-arrow) and then, manual evacuation of blood clots (B)

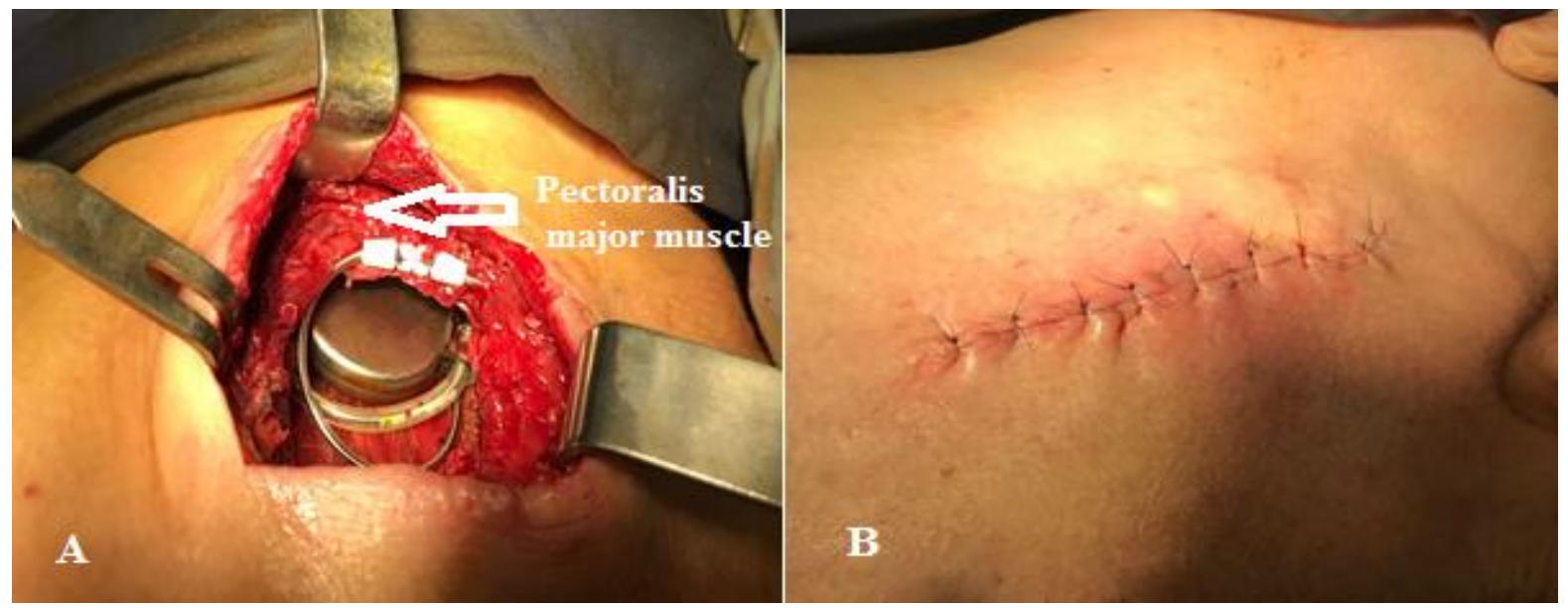

Fig. 7. Pectoralis major muscle-splitting approach to subpectoral place with generator insertion (A) and immediate closure after procedure (B)

Post-procedural, a chest X-ray confirmed the new location of the pacemaker (Figure 8).

In the next week, the patient continued antibiotherapy, being discharged from the hospital with recommendation of home treatment with Amoxicillin/ Clavulanic Acid 875 $\mathrm{mg} / 125 \mathrm{mg} \mathrm{q} 12 \mathrm{~h}$, Rilmenidine $1 \mathrm{mg}$ twice a day, Clopidogrel $75 \mathrm{mg}$ daily and Pantoprazole $40 \mathrm{mg}$ daily. Even though the patient had indication of anticoagulant treatment (the CHA2DS2-VASc score was 4), we opted for the antiplatelet therapy with Clopidogrel 
considering the patient non-compliance to treatment, and the risk of hematoma recurrence (a HAS BLED score of 4). We found out from his family that the patient had a favorable outcome, being expected for the next appointment.

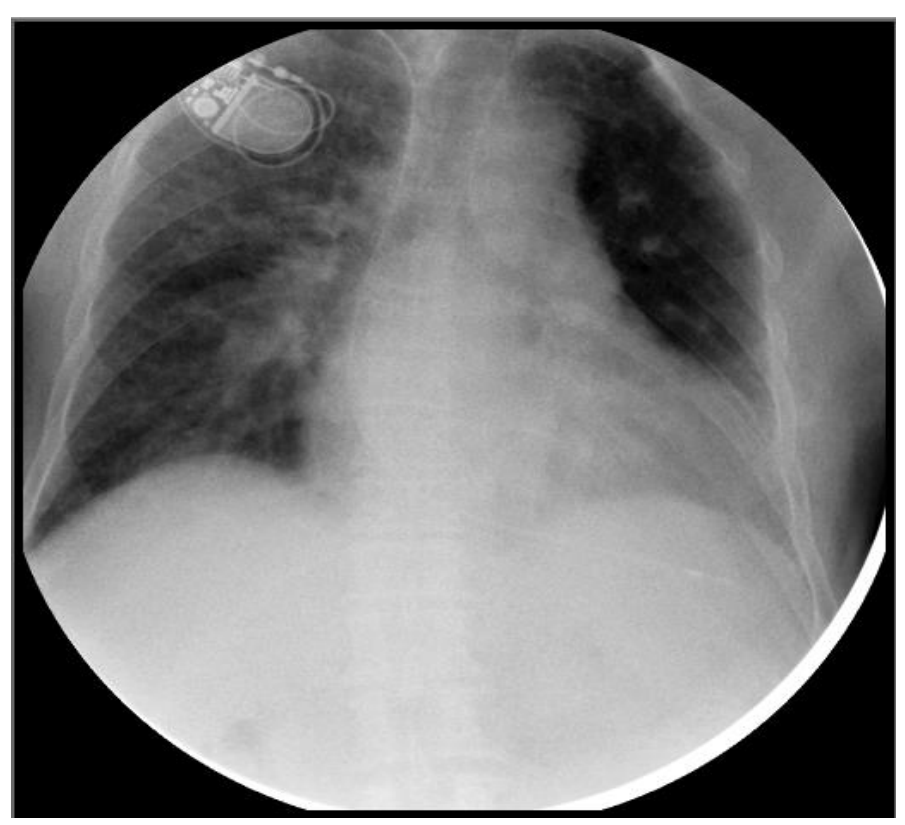

Fig. 8. Posteroanterior chest X-ray film showing the generator's new position

\section{Discussions}

The potential complications of implantable cardiac devices are significant, both in diversity and patient impact. The incidence of complications can be minimized with a clear understanding of the accepted implant indications and possible complications, and with a meticulous post-implant follow up.

The incidence of reported pacemaker system infection ranges from 2 to $8 \%$ [5]. Pacemaker pocket infection is an indication for complete device removal, with transvenous lead extraction. Under antimicrobial therapy, a new pacing system will be reimplanted [6]. Pavia $S$, et al presented the median time for device reimplantation, both pacing and ICD leads, to be of five days with no subsequent evidence of recurrent or new infection at a mean follow-up period of 46 weeks [7]. Device removal can cause devastating complications such as venous, valvular, or atrioventricular injury, tamponade, and sudden cardiac death $[8,9]$. So, in these cases, the risk associated with device extraction may outweigh that of attempted salvage surgery [10]. On the other hand, the mortality of persistent infection when infected leads are not removed can be as high as $66 \%$ [11]. In our case, advanced age, noncompliance, persisting pruritus and high risk of reinfection counterbalanced with the indication of pacing device removal. The salvage method was adopted, only after endocarditis and septicemia were excluded.

Eggimann, et al associated several factors with an increased risk of device infection, including diabetes mellitus, malignancy, corticosteroid use, anticoagulation, recent device manipulation, chronic renal failure, advanced age [12]. The patient presented some of these incriminating elements, but we assumed that the determining factor was represented by chronic pruritus. After excluding local and systemic causes of itch (cholestasis, uremia, hyperthyroidism, medications or lymphoma), the dermatologist established the diagnostic of idiopathic pruritus of the elderly or so-called Willian's itch [13].

Even though the patient received optimal dermatological treatment, his itching persisted, complicating even more the wound by developing pocket hematoma. This is an acute, relatively common complication, usually managed conservatively. Evacuation was 
reported in Kiviniemi, et al study as being required in 1 to $2 \%$ of implant cases [14]. In our case, the patient had indications for immediate drainage of blood clots (hematoma was expanding in size, being tense and painful).

In accordance with American and European Guidelines, the pacemaker implantation is consider as a minimally invasive procedure, without necessary of bridging therapy with heparin in the case of oral anticoagulated patient with low risk of bleeding $[15,16]$. In cases with intermediate risk (including patients with atrial fibrillation with CHA2DS2-VASc score of 4), it is recommended individually management for each case, considering procedure specific risks for bleeding and thromboembolism [17]. Due to infection appearance at pacemaker pocket, we preferred bridging therapy during hospitalization with low molecular weight heparin (Enoxaparin $60 \mathrm{mg}$ twice daily). After hematoma occurrence, LMWH was immediately interrupted, and after $24 \mathrm{~h}$ because the collection was expanding, it was decided to be evacuated. Twelve hours after hematoma evacuation we reinitiated Enoxaparin administration, but only one dose daily, until the day of pacemaker repositioning, when the dose of anticoagulant was skipped, and later until discharge.

Gold, et al demonstrated no significant differences in freedom from complications between subpectoral space device implant versus the traditional subcutaneous space demonstrated. Thus, cardiac electrophysiologists prefer to place these devices easily and quickly into the subcutaneous space, with shorter procedural time and no need of a plastic surgery consultation [18]. In case of infected or exposed cardiac devices, when device explantation is not feasible, salvage by repositioning often becomes imperative. Important advantages of the pectoralis major muscle splitting technique include maintenance of the original access incision, while safely placing the salvaged device into a fresh and well-vascularized tissue plan [1].
Even though the subpectoral positioning evolved without complications, we had information about his clinical course only from his family, without having the possibility to see his wound evolution and paraclinical parameters.

It is known that secondary procedures after an initial cardiac device implant represent a risk factor for infection, with a higher risk for early reinterventions in case of hematoma or lead dislodgement [19]. Because the reason for the second admission was the surgical site infection, followed by repeated reinterventions at the pacemaker pocket, it is necessary a long-term clinical, biological and echocardiographic follow-up for early detection of a possible endocarditis.

Allergic reactions to implantable cardiac device components are uncommon, and usually mimic a pacemaker infection. Hayes, et al, reported in 1997 an incidence of about 571 per 1 million of allergic sensitivity to pacemaker components [20]. Clinical presentation may be similar to pacemaker infection, but the allergy testing to various pacemaker components based on the manufacturer lot, it is highly specific for an allergic reaction diagnose. In this case, the patient complained of generalized pruritus at six months distance from initial implant, with secondary excoriations on his entire body, not only at the pacemaker pocket. Moreover, purulent secretions were present at the moment of admission at the surgical device site, and Staphylococcus aureus was revealed by the samples testing.

\section{Conclusions}

This is a particular case of pacemaker implant performed on strong indication, with favorable initial clinical course, but with complications (pocket infection and hematoma) occurring after six months, caused by an intrinsic factor (senile pruritus). Different items were analysed and subpectoral repositioning seemed to be the optimal conservative solution for this patient. 


\section{References}

1. Kistler PM, Fynn SP, Mond HG, Eizenberg N. The subpectoral pacemaker implant: it isn't what it seems! Pacing Clin Electrophysiol 2004; 27(3):361-364.

2. Knepp EK, Chopra K, Zahiri HR, Holton LH, Singh DP. An effective technique for salvage of cardiac-related devices. Eplasty 2012; 12: e8.

3. Gillette PC, Edgerton J, Kratz J, Zeigler V. The subpectoral pocket: the preferred implant site for pediatric pacemakers. Pacing Clin Electrophysiol 1991; 14(7):1089-1092.

4. Shimada $Y$, Matsukawa $M$, Yamamoto $F$. Subpectoral technique of pacemaker implantation- reduction of costs and length of hospital stay. J Rural Med 2008; 3(1):15-18.

5. Grimm W, Flores BF, Marchlinski FE, et al. Complications of implantable cardioverter defibrillator therapy: follow-up of 241 patients. PACE 1993, 16: 218-222.

6. Chua J, Wilkoff $B$, Lee I, et al. Diagnosis and management of infections involving implantable electrophysiologic cardiac devices. Ann Int Med 2000; 133:604-608.

7. Pavia S, Wilkoff B. The management of surgical complications of pacemaker and implantable cardioverter-defibrillators. Curr Opin Cardiol 2001; 16(1):66-71.

8. Brodman $R$, Frame $R$, Andrews $C$, et al. Removal of transvenous leads requiring cardiopulmonary bypass or inflow occlusion. $J$ Thorac Cardiovasc Surg 1992; 103:649-654.

9. Byrd CL. Advances in device lead extraction. Curr Cardiol Rep 2001; 3:324.

10. Kolker AR, Redstone JS, Tutela JP. Salvage of exposed implantable cardiac electrical devices and lead systems with pocket change and local flap coverage. Ann Plast Surg 2007; 59:26-30

11. Rettig G, Doenecke $P$, Sen $S$, et al. Complications with retained transvenous pacemaker electrodes. Am Heart J 1997; 98:587-594.

12. Eggimann $P$, Waldovogel FA. Pacemaker and defibrillator infections. In: Waldvogel FA, Bisno $A L$, eds. Infections associated with indwelling medical devices. Washington, DC: American Society for Microbiology Press, 2000:247.

13. Ward JR1, Bernhard JD. Willan's itch and other causes of pruritus in the elderly. Int $J$ Dermatol 2005; 44(4):267-273.

14. Kiviniemi $M$, Pirnes $M$, Eranen $H$, et al. Complications related to permanent pacemaker therapy. PACE 1999, 22:711-720.

15. January CT, Wann LS, Alpert JS, et al. 2014 AHA/ACC/HRS guideline for the management of patients with atrial fibrillation: $A$ report of the American College of Cardiology/ American Heart Association Task Force on Practice Guidelines and the Heart Rhythm Society. J Am Coll Cardiol 2014; 64:1-76.

16. Kristensen SD, Knuuti J, Saraste A, et al. 2014 ESC/ESA Guidelines on non-cardiac surgery: cardiovascular assessment and management. Eur Heart J 2014; 35:2383-2431.

17. Rechenmacher SJ, Fang JC. Bridging anticoagulation. J Am Coll Cardiol 2015; 66(12):1392-1403.

18. Gold MR, Peters RW, Johnson JW, et al. Complications associated with pectoral cardioverterdefibrillator implantation: comparison of subcutaneous and submuscular approaches. Worldwide Jewel Investigators. J Am Coll Cardiol 1996; 28:1278-1282.

19. Klug D, Balde M, Pavin D, et al. Risk Factors Related to Infections of Implanted Pacemakers and Cardioverter-Defibrillators. Circulation 2007; 116:1349-1355.

20. Hayes DL, Loesl K. Pacemaker component allergy: case report and review of the literature. Interv Card Electrophysiol 2002; 6(3):277-278. 